\section{EVALUACIÓN DE LA VULNERABILIDAD ANTE TSUNAMIS PARA EL SECTOR TURISMO EN VALPARAIISO, CHILE}

Manuel Contreras-López ${ }^{1, *}$, Patricia Araya ${ }^{2}$, Rodrigo Figueroa-Sterquel ${ }^{3}$, Wolfgang Alejandro Breuer ${ }^{4}$, Felipe Igualt ${ }^{5}$, Cristián Larraguibel-González ${ }^{3}$, Raúl Oberreuter ${ }^{6}$

\section{RESUMEN}

Se identificó y evaluó la vulnerabilidad de un total de 227 edificaciones asociadas al turismo en la ciudad puerto de Valparaíso, que se encuentran en el área de inundación definida para el peor escenario conocido para la zona. Se utilizó el Modelo de Evaluación de la Vulnerabilidad por Tsunami de Papathoma (PTVA-3). Los resultados muestran que las edificaciones asociadas al alojamiento, como hoteles, presentan bajos índices de vulnerabilidad, por lo que se recomienda considerar la evacuación vertical en estos casos. Las otras tipologías -alimentación, atractivos y serviciostambién presentan en general bajos índices de vulnerabilidad, pero con mayor dispersión. El puerto y la línea ferroviaria se han transformado en una barrera de protección y exclusión de emplazamientos de edificaciones en las zonas más expuestas. En los sectores emplazados entre la línea férrea y la línea costera, tales como Caleta Portales y Muelle Barón, se concentran las edificaciones con mayor índice de vulnerabilidad.

\section{PALABRAS CLAVE}

Evaluación vulnerabilidad por tsunami de Papathoma, PTVA-3, Vulnerabilidad urbana de edificaciones, Área de inundación

\section{ABSTRACT}

The vulnerability of a total of 227 buildings associated with tourism in the port city of Valparaíso, which is located in the flood area defined for the worst known scenario for the area, was identified and evaluated. The Papathoma Tsunami Vulnerability Assessment Model (PTVA-3) was used. The results show that the buildings were associated with accommodation, have low vulnerability indexes, it is recommended to consider vertical evacuation in these cases. The other typologies -restaurant, sight and services- also have low vulnerability indexes, but with greater dispersion. The port and the railway line have been transformed into a barrier of protection and exclusion of building sites in the most exposed areas. Sectors that remain outside the railway line, such as Caleta Portales and Muelle Barón, show the highest vulnerabilities.

\section{KEYWORDS}

Papathoma Tsunami Vulnerability Assessment, PTVA-3, Urban vulnerability of buildings, Flood area
1. Facultad de Ingeniería y Centro de Estudios Avanzados, Universidad de Playa Ancha, Valparaíso, Chile.

2. Programa Ingeniería Civil Oceánica, Universidad de Valparaíso, Valparaíso, Chile.

3. Instituto de Geografía, Pontificia Universidad Católica de Valparaíso, Valparaíso, Chile.

4. Facultad de Arquitectura y Diseño, Universidad Andrés Bello, Viña del Mar, Chile.

5. Escuela de Arquitectura y Diseño, Pontificia Universidad Católica de Valparaíso, Valparaíso, Chile.

6. Escuela de Ingeniería Civil en Obras Civiles, Facultad de Ingeniería y Ciencias, Universidad Diego Portales, Santiago, Chile.

*Autor de correspondencia: manuel.contreras@upla.cl

\section{RECIBIDO}

26 de septiembre de 2018

\section{ACEPTADO}

10 de diciembre de 2018

\section{PUBLICADO}

1 de enero de 2019

\section{Formato cita}

Recomendada (APA):

Contreras-López, M., Araya,

P., Figueroa-Sterquel, R., Breuer W.A., Igualt, F., Larraguibel-González, C. y Oberreuter, R. (2019). Evaluación de la vulnerabilidad ante tsunamis para el sector turismo en Valparaíso, Chile, Revista de Estudios Latinoamericanos sobre Reducción del Riesgo de Desastres REDER, 3(1), pp.5-23

\section{(ब) $(1) \Theta$}

Todos los artículos publicados en REDER siguen una política de Acceso Abierto y se respaldan en una Licencia CreativeCommons Atribución-NoComercial 4.0 Internacional.

\section{Revista de Estudios}

Latinoamericanos sobre Reducción del Riesgo de Desastres (REDER)

Diseño: Lupe Bezzina Tipografía: Hospital 


\section{INTRODUCCIÓN}

Valparaíso es la principal cuidad puerto de Chile y una de las más importantes del Pacífico Sur. Geográficamente, la ciudad se presenta en forma de un gran anfiteatro natural, emplazada en la bahía y rodeada de cerros, en los cuales vive la mayor parte de la población. Entre el pie de los cerros y el mar se forma "el plan", partes bajas e inundables donde se encuentra el centro administrativo, comercial y financiero de la ciudad. Posee una población que bordea los 300.000 habitantes (INE, 2017) y junto a Viña del Mar, conforma la conurbación costera más densamente poblada de Chile. Aquí se emplazan los principales ejes de comunicación interurbanos, servicios de emergencia, edificios de patrimonio histórico y cultural, establecimientos educaciones y universitarios, restaurantes, centros de entretención y capacidad hotelera.

Históricamente la ciudad nunca fue fundada, por lo que no posee un damero y funcionalmente es polinuclear. El lugar fue descubierto el 10 de abril de 1536 por Juan de Saavedra y designado como puerto natural de la ciudad de Santiago el 3 de septiembre de 1544 (Vicuña-Mackenna, 1869). Desde entonces, regularmente ha sido afectada por tsunamis, la mayoría no destructivos.

El primer registro de un tsunami destructivo en Valparaíso corresponde al sábado 8 de julio de 1730 (CERESIS, 1985). A las 01:45 (hora local), se produjo un terremoto mayor a 9,0 Mw (Carvajal et al., 2014) que fue percibido desde lquique hasta Osorno. Posteriormente, ondas de tsunami destructivo asolaron las partes bajas de la ciudad, alcanzando el templo de los padres de San Agustín y la iglesia de los Mercedarios (Carvajal et al., 2017a). En el barrio El Almendral, localizado al noreste del plan, todas las casas, fortificaciones y bodegas más inmediatas a la playa fueron destruidas por la inundación. Hasta el día de hoy no se ha repetido un tsunami de esa intensidad en la zona (Tabla 1). En esta misma tabla se puede apreciar que durante el siglo XIX ocurrieron seis sucesos generadores de tsunami, de los cuales el más importante fue el de 1822 y cuyo tsunami arrojó algunas embarcaciones menores a las puertas de la Aduana (Soloviev \& Go, 1984), sin provocar otros daños. Durante el siglo XX, de los 10 tsunamis que arribaron a Valparaíso, 8 fueron registrados instrumentalmente lo que facilita identificar eventos centimétricos que pasaban desapercibidos anteriormente. Ninguno de los tsunamis registrados durante el siglo XX y lo que va del siglo XXI han sido destructivos en Valparaíso. Así han pasado casi 300 años en que Valparaíso no ha experimentado un tsunami destructivo.

\begin{tabular}{|c|c|c|c|c|c|}
\hline $\begin{array}{l}\text { Fecha } \\
(A / M / D)\end{array}$ & Fuente & Origen & $\begin{array}{l}\text { Altura } \\
(\mathrm{m})\end{array}$ & Observación & Referencia \\
\hline $1730 / 07 / 08$ & Cercano & Sísmico Mw 9,0 & 12 & $\begin{array}{l}\text { Hora y día no laboral. } \\
\text { Tsunami destructivo. }\end{array}$ & Carvajal et al., 2017a \\
\hline $1811 / 11 / 19$ & Cercano & Sísmico & 4,0 & $\begin{array}{l}\text { Posiblemente tsunami no } \\
\text { fue destructivo. }\end{array}$ & Soloviev y Go, 1984 \\
\hline $1822 / 11 / 19$ & Cercano & Sísmico Mw 8,5 & 3,6 & Hora no laboral. & Soloviev y Go, 1984 \\
\hline $1835 / 02 / 20$ & Lejano & Sísmico Mw 8,2 & 0,5 & $\begin{array}{l}\text { En Valparaíso no fue } \\
\text { destructivo. }\end{array}$ & Soloviev y Go, 1984 \\
\hline $1868 / 10 / 16$ & Cercano & Sísmico & $<1$ & Hora no laboral. & Soloviev y Go, 1984 \\
\hline $1877 / 05 / 09$ & Lejano & Sísmico Mw 8,3 & 1,1 & Hora no laboral. & Soloviev y Go, 1984 \\
\hline $1877 / 09 / 02$ & Cercano & Meteorológico & Sin Dato & $\begin{array}{l}\text { Día no laboral. No se ha } \\
\text { confirmado origen }\end{array}$ & Goll, 1903 \\
\hline $1906 / 08 / 16$ & Cercano & Sísmico Mw 8,2 & 2,0 & $\begin{array}{l}\text { Hora no laboral. No fue } \\
\text { destructivo. }\end{array}$ & Soloviev y Go, 1984 \\
\hline $1943 / 04 / 06$ & Cercano & Sísmico Mw 8,2 & 0,8 & $\begin{array}{l}\text { En Valparaíso no fue } \\
\text { destructivo. }\end{array}$ & Soloviev y Go, 1984 \\
\hline $1946 / 04 / 01$ & Lejano & Sísmico Mw 8,6 & 0,8 & $\begin{array}{l}\text { En Valparaíso no fue } \\
\text { destructivo. }\end{array}$ & Shepard et al. 1949 \\
\hline $1952 / 11 / 04$ & Lejano & Sísmico Mw 9,0 & 0,9 & $\begin{array}{l}\text { En Valparaíso no fue } \\
\text { destructivo. }\end{array}$ & USDC, 1953 \\
\hline $1957 / 03 / 09$ & Lejano & Sísmico Mw 8,6 & 1,0 & Día no laboral. & Salsman, 1959 \\
\hline $1960 / 05 / 22$ & Lejano & Sísmico Mw 9,5 & 1,7 & Día no laboral. & SHOA, 2000 \\
\hline $1964 / 03 / 28$ & Lejano & Sísmico Mw 9,2 & 1,0 & Día no laboral. & $\begin{array}{l}\text { Spaeth y Berkman, } \\
1967\end{array}$ \\
\hline
\end{tabular}

(Continúa en la siguiente página) 
(Continuación)

\begin{tabular}{llllll}
$\begin{array}{l}\text { Fecha } \\
(\text { A/M/D) }\end{array}$ & Fuente & Origen & $\begin{array}{l}\text { Altura } \\
(\mathrm{m})\end{array}$ & Observación & Referencia \\
\hline $1971 / 07 / 09$ & Cercano & Sísmico Mw 7,8 & 1,2 & Hora no laboral. & NOAA, 2017 \\
\hline 1985/03/03 & Cercano & Sísmico Mw 8,0 & 1,2 & Hora y día no laboral. & NOAA, 2017 \\
\hline 1995/07/30 & Lejano & Sísmico Mw 8,0 & 0,3 & Hora y día no laboral. & NOAA, 2017 \\
\hline $2010 / 02 / 27$ & Cercano & Sísmico Mw 8,8 & 1,3 & Hora y día no laboral. & Fritz et al., 2011 \\
\hline $2011 / 03 / 11$ & Lejano & Sísmico Mw 9,1 & 1,5 & Hora y día no laboral. & NGDC, 2011 \\
\hline $2014 / 04 / 01$ & Lejano & Sísmico Mw 8,2 & 0,2 & Hora no laboral. & SHOA, 2014 \\
\hline $2015 / 08 / 15$ & Cercano & Meteorológico & 1,3 & Día no laboral. & Carvajal et al., 2017b \\
\hline $2015 / 09 / 16$ & Cercano & Sísmico Mw 8,3 & 2,2 & Hora no laboral. & Contreras-López et \\
\hline $2017 / 04 / 24$ & Cercano & Sísmico Mw 6,9 & 0,2 & No fue destructivo. & NOAA, 2017 \\
\hline
\end{tabular}

Tabla l. Cronología de tsunamis destructivos y no destructivos percibidos en Valparaíso, Chile Fuente: Contreras-López et al. (2019)

Entre las lecciones aprendidas del tsunami del oceánico índico de 2004 y del 27 febrero de Chile 2010, es que los turistas se encuentran entre el grupo de personas más vulnerables para este tipo de desastres (González \& Figueroa, 2005; Carlsen, 2006; Birkland et al., 2006; Contreras \& Winckler, 2013; Igualt et al., 2017). Se conjugan varios factores:

- La mejor respuesta para un tsunami de campo cercano es la auto-evacuación a sectores elevados. Esto es normalmente trasmitido por tradición oral entre los habitantes litorales, pero no necesariamente es conocido por turistas que pueden provenir de sectores interiores.

- El desconocimiento de áreas de inundación, vías de evacuación y los sectores de seguridad.

- Dependencia absoluta de los servicios de ayuda y respuesta a estas contingencias: La falta de redes de apoyo y todo el aislamiento que implica estar de paso por un lugar.

Actualmente el litoral central está sometido a una transformación humana intensiva donde sus actividades modifican el paisaje natural (Ranguel-Buitrago et al., 2018). La proximidad de Valparaíso a la Región Metropolitana de Santiago, donde habita el 40,4\% de la población del país (INE, 2017), genera una fuerte presión por diversos usos como el ocio y el turismo, reflejados en una alta inversión inmobiliaria organizada a lo largo de la costa. El desarrollo de resort o condominios multiplican la subdivisión predial mientras que la autoconstrucción de viviendas secundarias o de vacaciones incentivada por la construcción de infraestructura vial, estructuran una Macro Región Central de Chile que sirve de corredor de comercio bioceánico Atlántico-Pacífico.

La 'turistificación', es decir, la generalización espacial del turismo se produce, siguiendo a Knafou (1996) por la evolución compleja de tres fuentes: a) los turistas, a partir de su frecuentación; b) el mercado, a través de la puesta en venta de un producto previamente diseñado y; c) los planificadores y promotores del territorio, que relacionan iniciativas locales, regionales y nacionales a un espacio determinado.

Valparaíso es una ciudad-puerto con una relevante función de hospitalidad turística desde sus orígenes, lo que se ha profundizado en la actualidad. Algunos hitos de su historia lo demuestran:

- Alberga en 1663 el primer alojamiento formal del país y aquí se publica en 1883 el primer folleto turístico dirigido a forasteros (Canihuante, 2006).

- Su condición de destino recreacional, entre 1970 y 2000 se redujo a algunos espacios remanentes y marginales en el sur de la bahía.

- Desde 1910 la habilitación del Balneario de Las Torpederas.

- Hacia 1970 incorpora la caleta Portales y su extensa playa norte.

- Alberga el único Puerto deportivo de uso público de la macro-zona central del país (Puerto Deportivo de Valparaíso). 
- El proceso de reconversión productiva derivado de la deslocalización industrial y automatización de las faenas portuarias generó una importante intencionalidad del Estado por el turismo patrimonial, comercial e inmobiliario en el borde costero obsolescente de la función portuaria, donde destaca el proyecto de Mall Barón.

La coordinación estratégica entre el sector académico universitario, junto con la administración municipal y nacional y una acción tanto de cooperación como de oposición de la ciudadanía organizada, culminaron el año 2003 con el área histórica de Valparaíso, incluida en la Nómina del Patrimonio Mundial por la UNESCO, por constituir un testimonio de la globalización durante el fin del siglo XIX, volviéndose el primer puerto del Océano Pacífico y recibiendo influencias económicas, sociales y culturales durante varias migraciones. La arquitectura y su matriz natural dan a Valparaíso un carácter único que justifica su presencia en aquella lista. Desde la declaración, se ha observado un crecimiento del turismo local y cambios espaciales notables, la zona histórica transformándose en un barrio privilegiado para los turistas y las actividades de ocio, culturales y de consumo, cerrando un ciclo demográfico y migratorio que vacía de actividad residencial al área desde el terremoto de 1985.

Así el fenómeno turístico recibió un impulso, implicando un aumento en el número de visitantes de la ciudad que se concentran durante el verano los meses de diciembre a marzo, luego regresan en las vacaciones escolares invernales en julio, retornan en las fiestas patrias de septiembre, salpicando la costa y la ciudad con breves visitas los fines de semana largos por festividades cívicas, militares y religiosas del calendario festivo chileno. Al mismo tiempo, la población de Valparaíso se ha encontrado con un crecimiento acotado, inferior al crecimiento nacional y del resto de las comunas costeras del país (Tabla 2). Sólo en los últimos 5 años se ha experimentado un crecimiento que supera marginalmente el estancado crecimiento poblacional nacional, debido a la abrupta disminución de las tasas de natalidad.

\begin{tabular}{llllll} 
& Censo 1982 & Censo 1992 & Censo 2002 & $\begin{array}{l}\text { Población } \\
\text { (estimada 2012) }\end{array}$ & Censo 2017 \\
\hline Valparaíso & 272.920 & 282.840 & 275.982 & 294.023 & 296.655 \\
\hline Comunas costeras & 289.9231 & 347.7241 & 3.953 .562 & 4.534 .212 & 4.534 .938 \\
\hline Total nacional & 11.329 .736 & 13.348 .401 & 15.116 .435 & 17.444 .799 & 17.574 .003 \\
\hline \% Variación Valparaíso & & 3,63 & $-2,42$ & 6,54 & 0,90 \\
\hline \% Variación comunas costeras & & 19,94 & 13,70 & 14,69 & 0,02 \\
\hline \% Variación nacional & & 17,82 & 13,25 & 15,40 & 0,74 \\
\hline
\end{tabular}

Tabla 2. Variación del número de habitantes de la comuna de Valparaíso, el total de comunas costeras de país y el total nacional, según los censos 1982 - 2017

Fuente: Instituto Nacional de Estadística, Chile

Desafortunadamente no existen estadísticas a escala local sobre las visitas turísticas. Las existentes mantienen sesgos importantes, lo que las convierte en inútiles para conocer el crecimiento del turismo en la comuna Valparaíso. Sin embargo, es posible estimar la estacionalidad y vislumbrar la existencia de un crecimiento constante en base a estadísticas indirectas: a) el número de viajes por automóvil entre Valparaíso y Santiago, y b) la cantidad de pasajeros provenientes de cruceros que desembarcan en el puerto.

La Figura 1 muestra la diferencia porcentual de viajes entre meses del año durante la presente década en la carretera que une a Valparaíso con Santiago, principal origen de los visitantes al puerto, sobre una base de 25,7 millones de viajes en 2010 a 40,1 millones de viajes en 2017, demostrando el aumento de pulsos de uso en las fechas indicadas precedentemente (época estival, vacaciones de invierno y algunas festividades). Obviamente no todos los viajes se realizan por turismo ni todos llegan como destino a la ciudad puerto, pero una parte importante lo hace.

Un fenómeno inédito corresponde al desarrollo de una industria de cruceros internacional que realiza una temporada de recaladas en Valparaíso en los últimos diez años, impulsado por la estrategia turística cultural de los actores territoriales. Implica la instalación de infraestructura habilitante para los controles de pasajeros y equipaje internacional y el transporte desde el barco 
a la ciudad. Esta localización es en la orilla occidental de la ciudad-puerto, entre la línea de costa y la línea del ferrocarril, que actúa como barrera. Esto ha marcado la internacionalización del destino Valparaíso Patrimonio de la Humanidad con recaladas en aumento que implican la visita por pocas horas desde unos 10.000 a 100.000 pasajeros extranjeros (ver Figura 2).

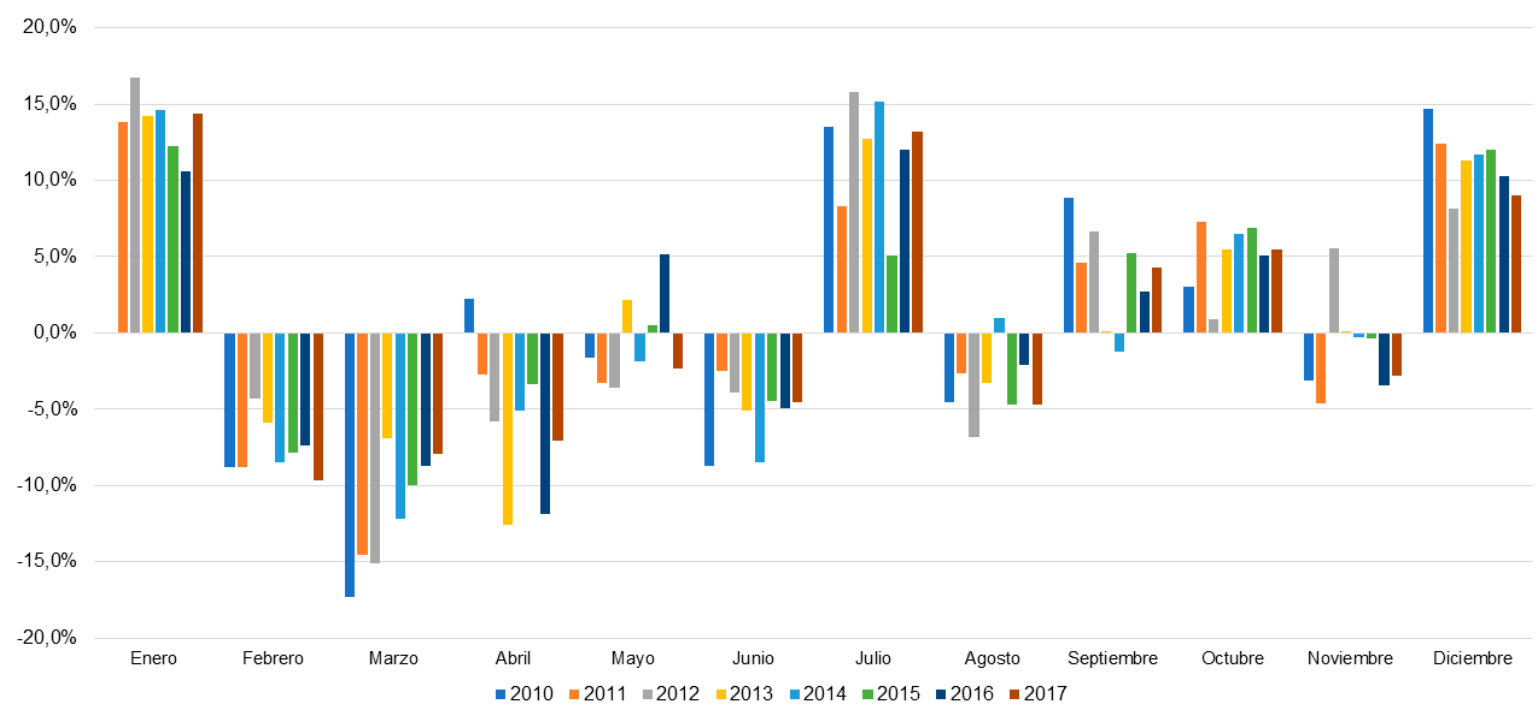

Figura 1. Variación mensual de los viajes entre el eje vial Santiago - Valparaíso durante: 2010 - 2017 Fuente: Autores, 2019, en base a MOP (2018)

Sabiendo que el turismo en Valparaíso ha experimentado un importante crecimiento en las últimas décadas, que existen ciertas fechas en el año en que la cantidad de visitantes crece exponencialmente y que Valparaíso es un lugar amenazado por la ocurrencia de tsunamis, el objetivo de este trabajo es evaluar la vulnerabilidad frente a tsunamis de la infraestructura asociada al sector turismo en la ciudad de Valparaíso. Para realizar esta evaluación se utiliza la metodología propuesta por Papathoma que se describe en la siguiente sección. Estudios similares se han realizado en Grecia (Papathoma \& Dominey-Howes, 2003), Italia (Alberico et al., 2015), Maldivas (DomineyHowes \& Papathoma, 2007), y Australia (Dall'Osso et al., 2016). En Chile se han efectuado este tipo de estudios en Concón (Igualt, 2017) y en Coquimbo - La Serena (Fritis et al., 2018).

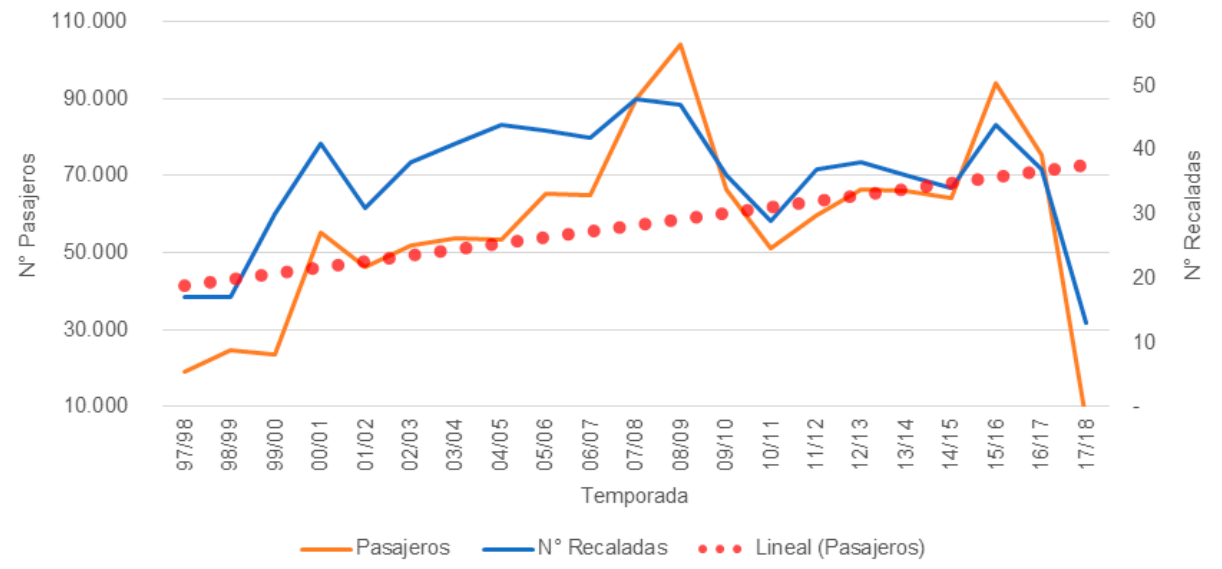

Figura 2. Evolución del número de pasajeros y recaladas de cruceros en el puerto de Valparaíso en las temporadas

estivales de 1997 a 2018
Fuente: Empresa Portuaria Valparaíso 


\section{METOdOLOGÍA}

Se utilizó Sightsmap para identificar las zonas de mayor interés turístico en Valparaíso, de acuerdo con la metodología propuesta por Tammet et al. (2013), que se sustenta en la valorización indirecta que realizan los visitantes sobre puntos y áreas particulares en sus visitas turísticas. Utilizando los teléfonos móviles y conectados a Internet dan cuenta y comparten sus preferencias y prácticas espaciales a través de fotografías. Tammet et al. (2013) analizan y combinan una serie de bases de datos georreferenciadas mundiales con el objetivo de localizar, describir y clasificar los destinos turísticos potenciales en cualquier área del mundo, utilizando las fotografías que los propios visitantes han capturado y compartido a través de la red social Panoramio y las ha mapeado en Sightsmap generando puntos calor de mayor densidad de concentración de fotografías.

El área de estudio comprende el área de inundación por tsunami de Valparaíso (Figura 3), señalada en la carta TSU-5110 Valparaíso - Viña del Mar (SHOA, 2012), referida al evento de 1730, que actualmente se considera el peor escenario de inundación para la zona. Esta área se recorrió a pie entre los días 30 de julio y 7 de agosto de 2018; con un equipo GPS Garmin 64, una cámara fotográfica y un plano de ubicación con el área de inundación estimada.

El área de estudio se recorrió por sectores y se buscaron los siguientes tipos de establecimientos: a) alojamiento formal (hoteles, hostales, residenciales) e informal (arriendo de habitaciones); b) alimentación (restaurantes, comida al paso, fuentes de soda, cafeterías, gelaterías); c) atractivos turísticos (patrimoniales, galerías artesanales, souvenir) y de entretención (bar-pubs, cine, actividades náuticas); y d) servicios financieros (bancos, cajeros automáticos, casas de cambio), transporte (terminal de pasajeros, estaciones de traslado) y de comunicación (centros de llamados e internet, correos). En la Tabla 3, se da a conocer la cantidad y tipo de establecimientos emplazados en la zona de inundación. Se encontró un total de 271 establecimientos, distribuidos en 227 edificios evaluados (en un edificio puede haber más de un establecimiento).
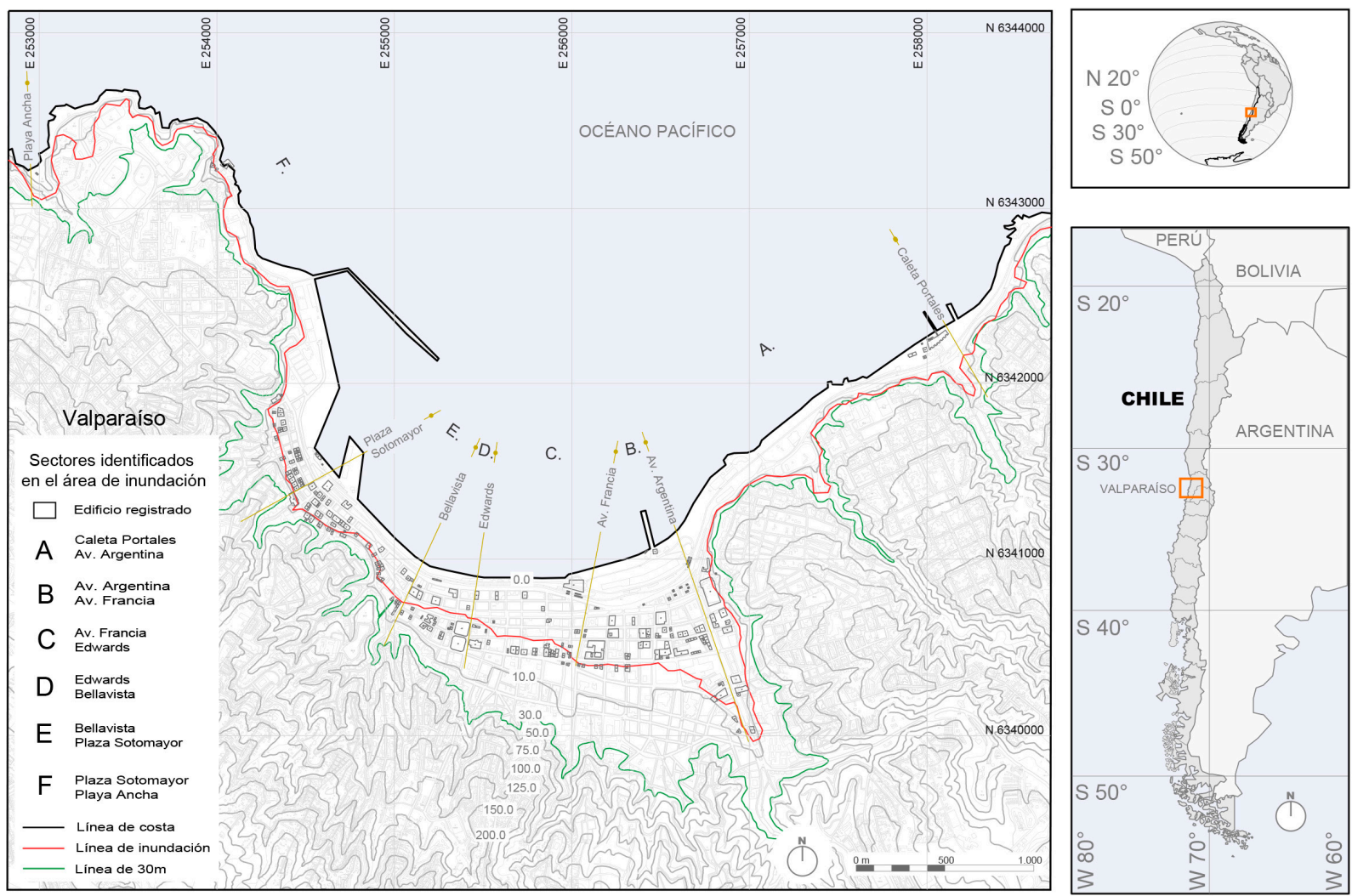

Figura 3. Área de Estudio ciudad puerto de Valparaíso. La línea roja señala la línea de inundación por tsunami. Los recuadros

grises señalan las edificaciones del sector turismo prospectadas
Fuente: Autores, 2019 


\begin{tabular}{l|l|l|l|l|l|l|l|} 
& \multicolumn{7}{|c|}{ Sectores identificados en el área de inundación } \\
\cline { 2 - 9 } & A & B & C & D & E & F \\
\cline { 2 - 9 } & $\begin{array}{l}\text { Caleta Portales } \\
\text { hasta Av. } \\
\text { Argentina }\end{array}$ & $\begin{array}{l}\text { Av. Argentina } \\
\text { hasta Av. } \\
\text { Francia } \\
\text { establecimiento }\end{array}$ & $\begin{array}{l}\text { Av. Francia hasta } \\
\text { Edwards }\end{array}$ & $\begin{array}{l}\text { Edwards hasta } \\
\text { Bellavista }\end{array}$ & $\begin{array}{l}\text { Bellavista hasta } \\
\text { Plaza Sotomayor }\end{array}$ & $\begin{array}{l}\text { Plaza Sotomayor } \\
\text { hasta Playa } \\
\text { Ancha }\end{array}$ & Total \\
\hline Alojamiento & 4 & 13 & 2 & 5 & 1 & 3 & 28 \\
\hline Alimentación & 10 & 33 & 19 & 10 & 40 & 37 & 149 \\
\hline Atractivos Turísticos & 5 & 5 & 8 & 8 & 11 & 13 & 50 \\
\hline Servicios & 3 & 11 & 4 & 6 & 19 & 1 & 44 \\
\hline No Establecimientos & 22 & 62 & 33 & 29 & 71 & 54 & 271 \\
\hline
\end{tabular}

Tabla 3. Tipos y cantidad de establecimientos identificados en el área de inundación de la ciudad de Valparaíso Fuente: Autores, 2019

Cada vez que se identificó alguno de estos establecimientos, se procedió a georreferenciarlo, fotografiarlo y evaluar su vulnerabilidad de acuerdo con la metodología propuesta por Papathoma versión 3: PTVA-3 (Dall'Osso et al., 2009), que se detalla en la Tabla 4 y 5.

\begin{tabular}{|c|c|c|c|c|c|c|c|}
\hline \multirow[b]{2}{*}{ Atributo } & \multicolumn{7}{|c|}{ Índices } \\
\hline & -1 & $-0,5$ & 0 & $+0,25$ & $+0,5$ & $+0,75$ & +1 \\
\hline Número de pisos (s) & Más de 5 pisos & 4 pisos & 3 pisos & & 2 pisos & & 1 piso \\
\hline Materialidad (m) & $\begin{array}{l}\text { Hormigón } \\
\text { armado }\end{array}$ & & $\begin{array}{l}\text { Doble ladrillo } \\
\text { (Albañilería } \\
\text { reforzada) }\end{array}$ & & $\begin{array}{l}\text { Ladrillo simple } \\
\text { (Albañilería) }\end{array}$ & Adobe & Madera \\
\hline $\begin{array}{l}\text { Hidrodinámica del } \\
\text { primer piso (g) }\end{array}$ & Planta abierta & $\begin{array}{l}\text { Planta abierta } \\
\text { con ventanas }\end{array}$ & $\begin{array}{l}\text { Planta abierta } \\
50 \%\end{array}$ & & $\begin{array}{l}\text { Planta cerrada con } \\
\text { muchas ventanas }\end{array}$ & & Planta cerrada \\
\hline $\begin{array}{l}\text { Resistencia de la } \\
\text { fundación (f) }\end{array}$ & $\begin{array}{l}\text { Fundación } \\
\text { Profunda }\end{array}$ & & $\begin{array}{l}\text { Fundación } \\
\text { promedio }\end{array}$ & & & & $\begin{array}{l}\text { Fundación } \\
\text { superficial }\end{array}$ \\
\hline $\begin{array}{l}\text { Forma y orientación } \\
\text { (so) }\end{array}$ & $\begin{array}{l}\text { Forma } 100 \% \\
\text { hidrodinámica }\end{array}$ & & $\begin{array}{l}\text { Forma 50\% } \\
\text { hidrodinámica }\end{array}$ & & & & $\begin{array}{l}\text { Forma poco } \\
\text { hidrodinámica }\end{array}$ \\
\hline $\begin{array}{l}\text { Objetos móviles } \\
\text { (mo) }\end{array}$ & & & $\begin{array}{l}\text { Riesgo mínimo } \\
\text { de daño por } \\
\text { objetos móviles }\end{array}$ & $\begin{array}{l}\text { Riesgo modera- } \\
\text { do de daño por } \\
\text { objetos móviles }\end{array}$ & $\begin{array}{l}\text { Riesgo promedio } \\
\text { de daño por obje- } \\
\text { tos móviles }\end{array}$ & $\begin{array}{l}\text { Riesgo alto de } \\
\text { daño por obje- } \\
\text { tos móviles }\end{array}$ & $\begin{array}{l}\text { Riesgo extremo } \\
\text { de daño por } \\
\text { objetos móviles }\end{array}$ \\
\hline $\begin{array}{l}\text { Condición de con- } \\
\text { servación (pc) }\end{array}$ & Muy bueno & Bueno & Promedio & & Malo & & Muy Pobre \\
\hline
\end{tabular}

Tabla 4. Atributos y valores que determinan la vulnerabilidad estructural de la construcción ( $B v)$. Valores positivos indican un incremento en la vulnerabilidad, mientras que los valores negativos indican un decrecimiento Fuente: Adaptado de Dall'Osso et al. (2009)

\begin{tabular}{|c|c|c|c|c|c|}
\hline \multirow[b]{2}{*}{ Atributo } & \multicolumn{5}{|c|}{ Índices } \\
\hline & 0 & $+0,25$ & $+0,5$ & $+0,75$ & +1 \\
\hline $\begin{array}{l}\text { Número de Fila } \\
\text { (Prot br) }\end{array}$ & $>10^{\circ}$ & $7-8-9-10^{\circ}$ & $4-5-6^{\circ}$ & $2-3^{\circ}$ & $1^{\circ}$ \\
\hline $\begin{array}{l}\text { Barreras naturales } \\
\text { (Prot } \mathrm{nb})\end{array}$ & Protección muy alta & Protección alta & Protección promedio & Protección moderada & Sin protección \\
\hline $\begin{array}{l}\text { Forma y altura de } \\
\text { malecón (Prot sw) }\end{array}$ & Vertical y >5[m] & Vertical y de 3 a 5 [m] & Vertical y de 1,5 a $3[\mathrm{~m}]$ & $\begin{array}{l}\text { Vertical y de } 0 \text { a } 1,5[\mathrm{~m}] \text { ó } \\
\text { inclinado y de } 1,5 \text { a } 3[\mathrm{~m}]\end{array}$ & $\begin{array}{l}\text { Inclinado y de } 0 \text { a 1,5 } \\
\text { [m] ó sin protección }\end{array}$ \\
\hline $\begin{array}{l}\text { Muro alrededor del } \\
\text { edificio (Prot w) }\end{array}$ & $\begin{array}{l}\text { La altura de la pared es } \\
\text { del } 80 \% \text { al } 100 \% \text { de la } \\
\text { profundidad del agua }\end{array}$ & $\begin{array}{l}\text { La altura de la pared } \\
\text { es del } 60 \% \text { al } 80 \% \text { de la } \\
\text { profundidad del agua }\end{array}$ & $\begin{array}{l}\text { La altura de la pared } \\
\text { es del } 40 \% \text { al } 60 \% \text { de la } \\
\text { profundidad del agua }\end{array}$ & $\begin{array}{l}\text { La altura de la pared } \\
\text { es del } 20 \% \text { al } 40 \% \text { de la } \\
\text { profundidad del agua }\end{array}$ & $\begin{array}{l}\text { La altura de la pared } \\
\text { es de } 0 \% \text { a } 20 \% \text { de la } \\
\text { profundidad del agua }\end{array}$ \\
\hline
\end{tabular}

Tabla 5. Valores asignados a los cuatro atributos que determinan la protección del edificio (Prot). Valores cercanos a 0 indican un alto nivel de protección, mientras que valores cercanos a 1 indican un bajo nivel de protección

Fuente: Adaptado de Dall'Osso et al. (2009) 
resultados muestran al sector de la Caleta Portales como la zona más vulnerable del equipamiento turístico de Valparaíso. En parte, su vulnerabilidad se explica por ser uno de los pocos sectores de Valparaíso cuya infraestructura está emplazada contigua a la línea de costa. Esta situación es diferente en las otras zonas de Valparaíso, donde la ocupación contigua a la línea de costa es utilizada por actividades portuarias y la línea férrea de MERVAL (Metro Valparaíso), la que se extiende por todo el borde de la ciudad de Valparaíso hasta la plaza Sotomayor en el sector Puerto. Por este motivo se plantea la necesidad de contar con una planificación especial para esta zona de alta vulnerabilidad, debido a que es una zona de alta demanda productiva y de gran conectividad para la movilidad de la población que reside en las zonas del interior de la región.

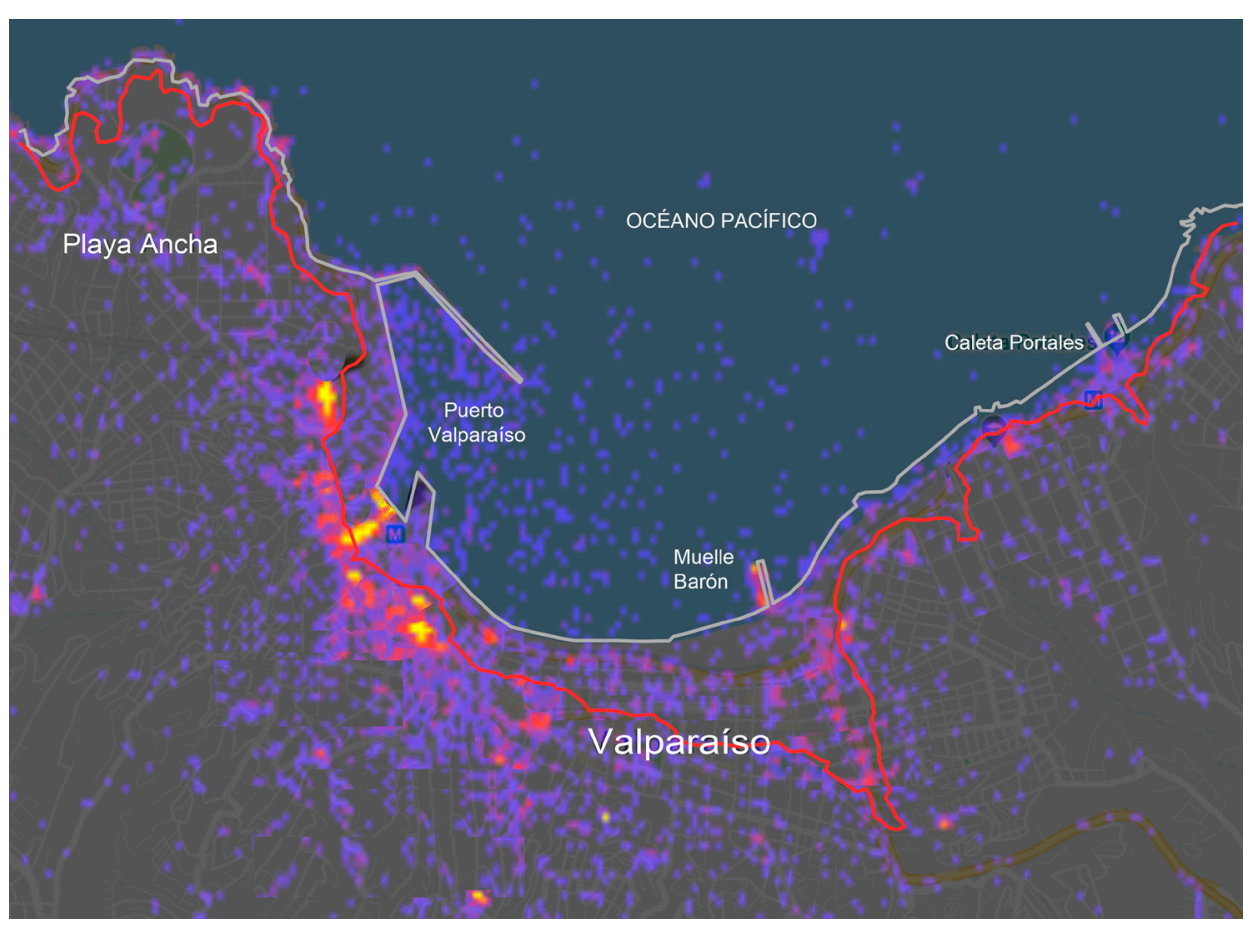

Figura 4. Puntos de interés turístico en la ciudad de Valparaíso y línea de inundación por tsunami Fuente: Adaptado de Sightsmap (2018)

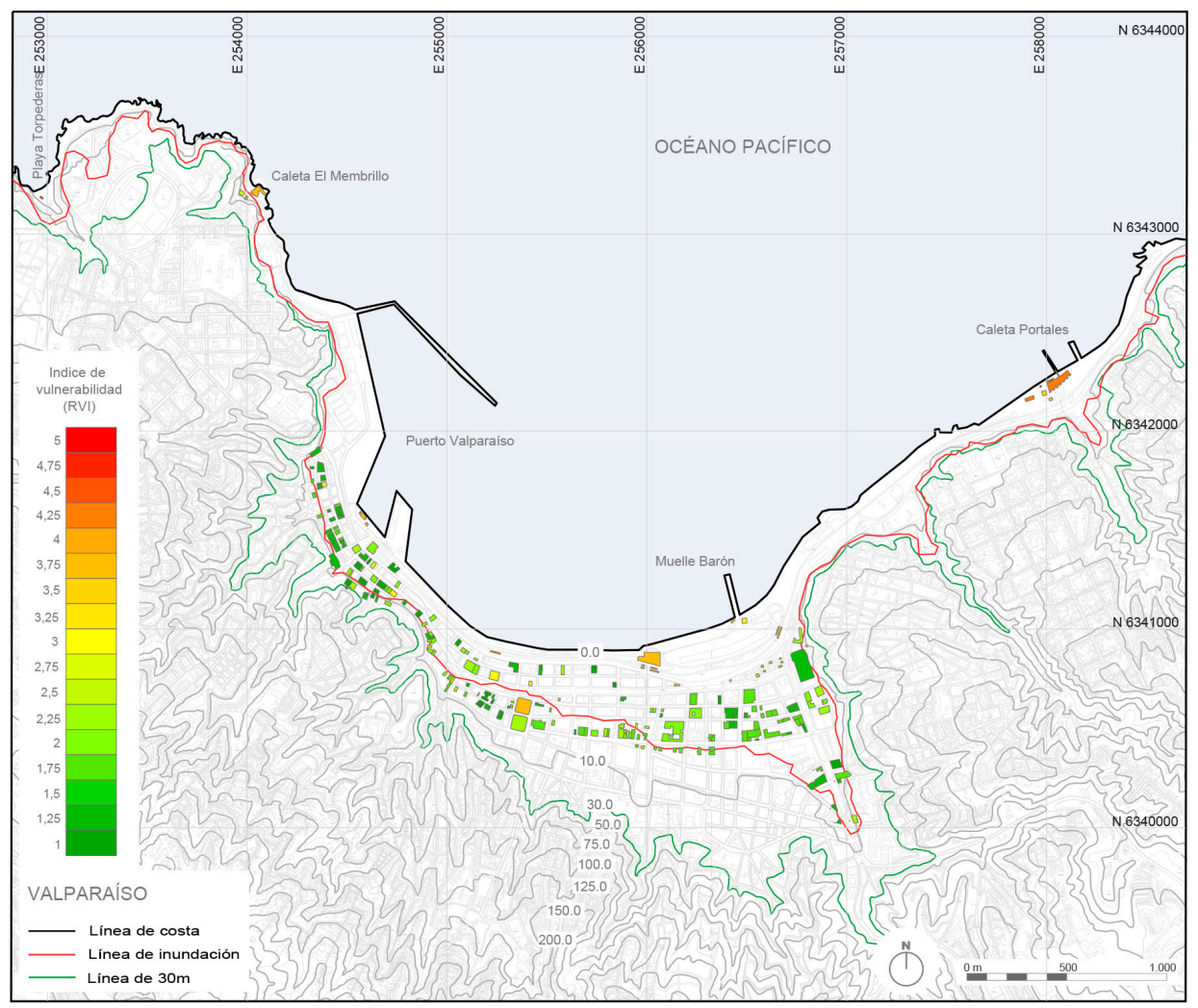

Figura 5. Distribución espacial de los Índices de Vulnerabilidad Relativa (RVI) para edificaciones asociadas al turismo, de acuerdo a Papathoma (PTVA-3) para el área de inundación por tsunami en Valparaíso Fuente: Autores, 2019 
En la Tabla 7, se muestran estadígrafos para el total de edificaciones estudiadas ( $\mathrm{N}=227)$ y segregado por sectores (alojamiento, alimentación, atractivos y servicios).

\begin{tabular}{l|l|l|l|l|l} 
Estadígrafos & Alimentación & Alojamiento & Atractivos & Servicios & TOTAL \\
\hline $\mathrm{N}$ & 121 & 25 & 42 & 39 & 227 \\
\hline$\%$ & 53,30 & 11,01 & 18,50 & 17,18 & 100,00 \\
\hline Media & 1,73 & 1,68 & 1,96 & 2,02 & 1,89 \\
\hline Media Geométrica & 1,85 & 1,63 & 1,83 & 1,80 & 1,76 \\
\hline Mínimo & 1,00 & 1,00 & 1,00 & 1,00 & 1,00 \\
\hline Máximo & 5,00 & 2,33 & 4,33 & 4,33 & 5,00 \\
\hline Desviación Estándar & 0,79 & 0,42 & 0,82 & 0,98 & 0,81 \\
\hline Asimetría & 2,23 & 0,05 & 1,60 & 0,73 & 1,71 \\
\hline Curtosis & 5,88 & $-0,93$ & 2,13 & $-0,73$ & 3,11 \\
\hline Coeficiente Variación & 45,80 & 25,28 & 41,85 & 48,69 & 42,96 \\
\hline
\end{tabular}

Tabla 7. Estadígrafos de los índices de Vulnerabilidad por tsunami para edificaciones asociadas al turismo en Valparaíso, segregados por áreas Fuente: Autores, 2019

\section{Alojamiento}

Se encontraron un total de 25 edificios asociados al alojamiento de turistas en el área de inundación, los que se dividen en dos grupos: alojamientos formales (hoteles, hostales, residenciales) e informales (arriendo de habitaciones). En general muestran un bajo índice de vulnerabilidad, con valores que varían en un rango de 1 a 2 (menos vulnerables a sufrir daño ante una inundación por tsunami), situación que se justifica por el grado de exposición a la profundidad del agua, menor a 1 [m] para todas las edificaciones que prestan este tipo de servicio y a los atributos estructurales de cada construcción.

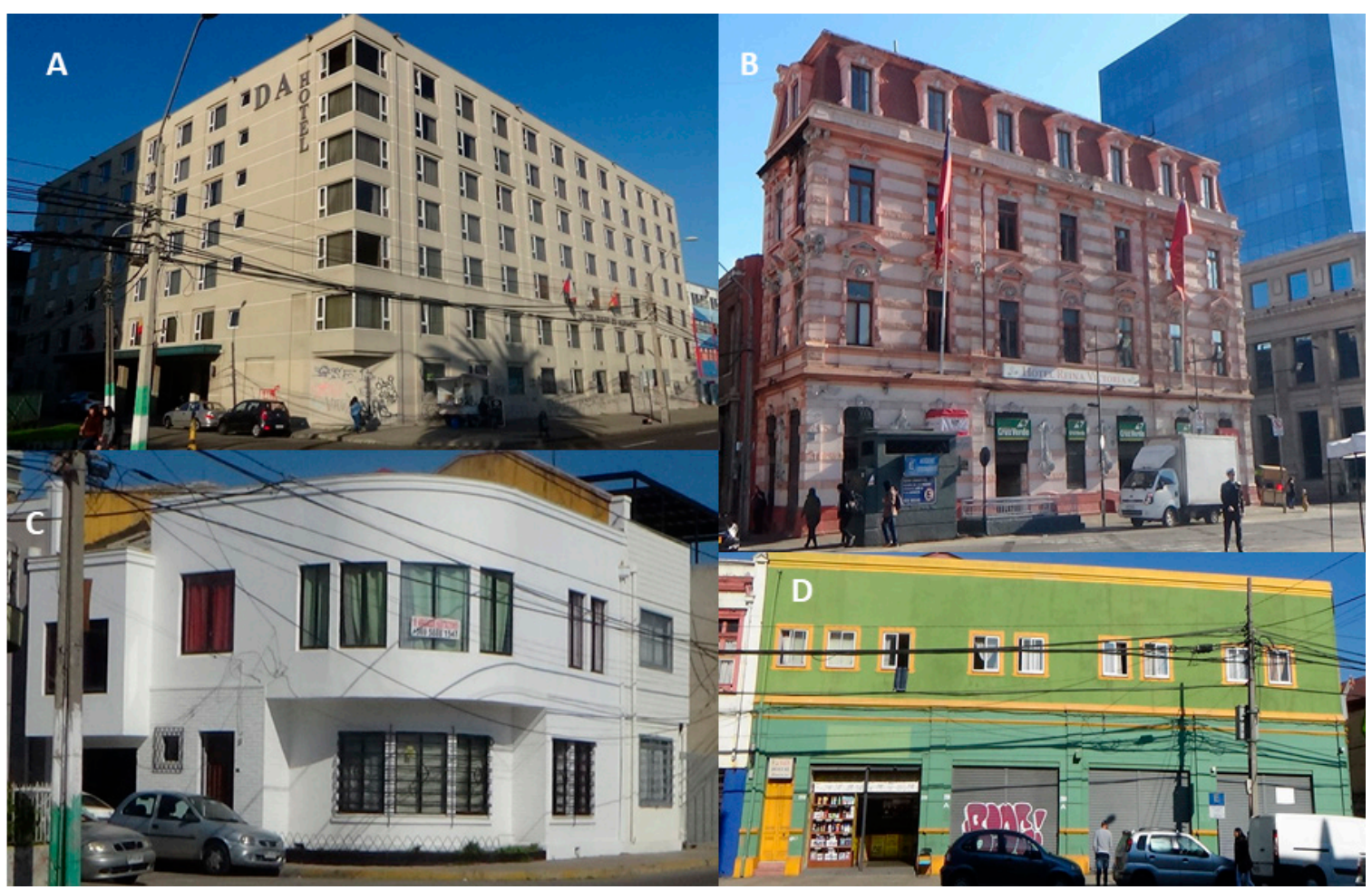

Figura 6. Ejemplos de edificaciones asociadas al alojamiento de turistas en el área de inundación por tsunami en

Valparaíso. A) Hotel de construcción moderna y robusta, en un área altamente expuesta, pero con baja vulnerabilidad

por tsunami. B) Hotel de construcción antigua, pero que también muestra una baja vulnerabilidad por tsunami. C)

Alojamiento informal con estructura robusta emplazado en una zona con gran exposición a una inundación por tsunami.

D) Ejemplo alojamiento informal de una estructura vulnerable pero emplazado en un área con baja inundación potencial

Fuente: Autores, 2019 
En los alojamientos formales las características que prevalecen corresponden a edificios de dos o más pisos, que tienen una materialidad y sistema constructivos robustos, siendo los hoteles los que presentan los índices más bajos (RVI =1), edificios que se caracterizan por ser construidos de hormigón armado y con fundaciones profundas, tienen más de 4 niveles y se encuentran en muy buen estado de conservación (Figura 6 A y B), a pesar de que el entorno ofrece un nivel bajo de protección y la exposición a los objetos móviles es relativamente alta. En cuanto a los alojamientos informales, estos son más pequeños, pero de construcción robusta, principalmente de hormigón. Sin embargo, los edificios que presentaron un índice de vulnerabilidad más elevado ( $R V I=2,3$ ), cuentan con atributos estructurales más desfavorables, son construcciones de adobe o albañilería simple y tienen menos niveles, pero nunca tienen una sola planta (Figura 6 C y D).

\section{Alimentación}

Se encuestaron 121 edificaciones correspondientes al ítem alimentación. Estos presentan una distribución homogénea en la zona de inundación, presentando una amplia variedad de tipologías constructivas (ver Figura 7).

Los establecimientos que prestan servicios de alimentación (restaurantes, comida al paso, fuente de soda, cafés y similares), arrojaron índices de vulnerabilidad bajos, valores que varían en un rango de 1,3 a 2,3 lo que implica que son menos vulnerables a sufrir daño ante una inundación por tsunami, situación que se justifica por el grado de exposición a la profundidad del agua, menor a 1 [m] y a las características estructurales de cada edificación, las que resultaron ser aceptables $(\mathrm{Bv}=1-3)$ para la mayoría de ellas, a pesar de que el entorno ofrece un nivel bajo de protección (Prot $=3-5)$.

Sin embargo, los recintos que presentan un índice de vulnerabilidad alto son los que están expuestos a profundidades mayores de inundación y presentan atributos estructurales de construcción desfavorables, los que se caracterizan principalmente por ser construidos con materiales ligeros, y aunque tienen plantas abiertas en el primer nivel, han sido fundadas superficialmente y el riesgo de recibir algún tipo de daño por objetos móviles es alto.

Se observa que los locales de alimentación principalmente ocupan los primeros pisos de edificios. Esto se explica en la necesidad de accesibilidad peatonal, sin embargo, es una de las características que incrementa la vulnerabilidad de estos. Además, se aprecia una mayor cantidad de construcciones en material ligero que en otros ítems. Otra característica es la presencia de más ventanales o aberturas del primer nivel que en otros ítems, fundamentado en ofrecer vistas a los usuarios, lo que permitiría la circulación del agua por el primer nivel durante una inundación.

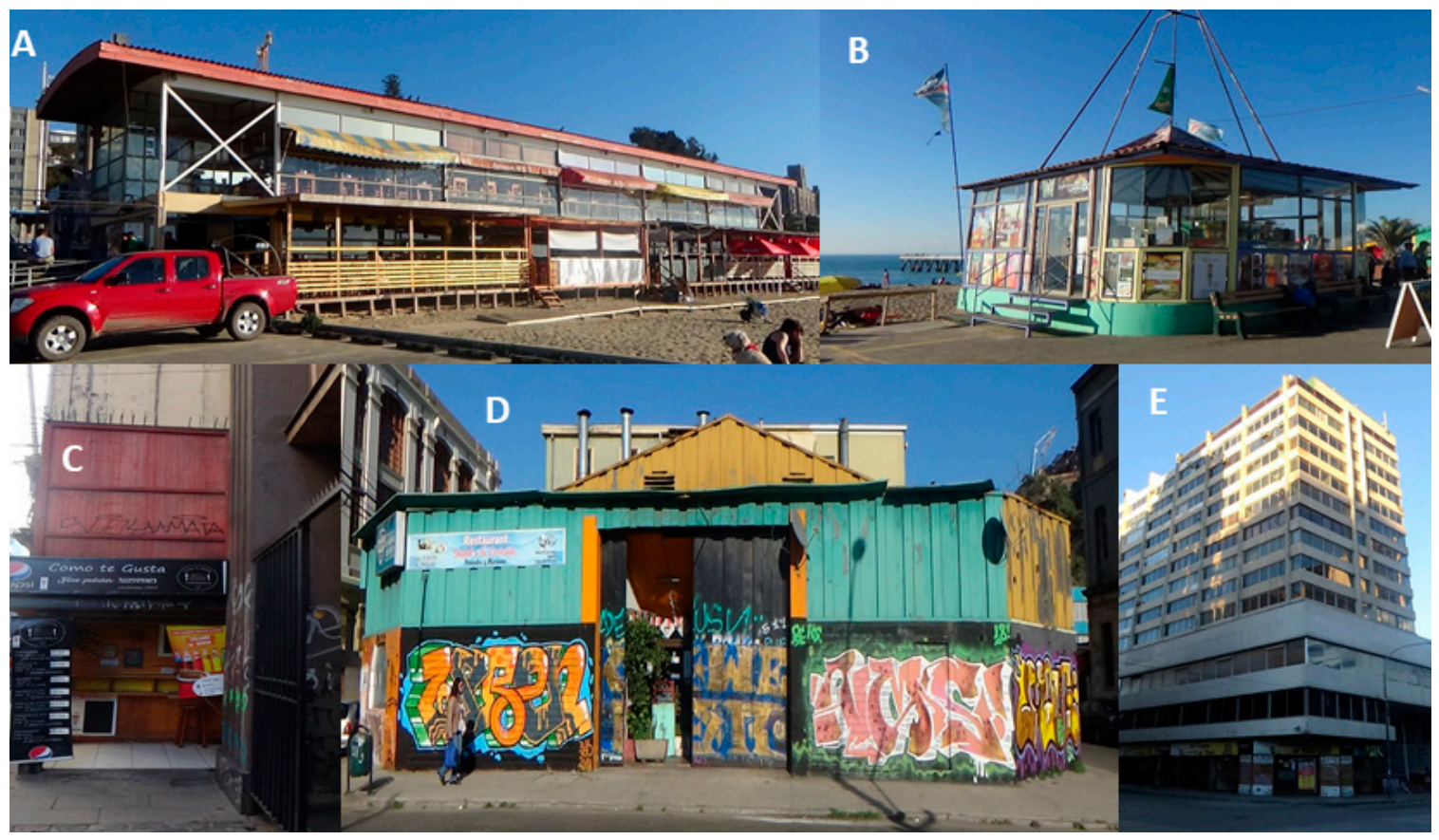

Figura 7. Ejemplos de edificaciones asociadas al ítem alimentación del sector turismo. A) Ejemplo estructuras altamente vulnerables a una inundación por tsunami en el sector caleta Portales. B) ejemplo estructura con vulnerabilidad muy alta. C) y D) estructura con vulnerabilidad promedio. E) edificación con vulnerabilidad baja Fuente: Autores, 2019 


\section{Atractivos turísticos}

Respecto a las 42 edificaciones de atractivos turísticos encuestados, estos se emplazan principalmente en el Sector Puerto y Bellavista, en edificios antiguos pero robustos y en un buen estado de preservación (ver Figura 8). Estos tienen menos dispersión que los de alimentación y también se parecen mucho en tipología.

Los establecimiento que se caracterizan por ser atractivos turísticos como edificios patrimoniales, comerciales, centros recreativos, entre otros, arrojaron índices de vulnerabilidad bajos $(\mathrm{RVI}=1-2,3)$, lo que implica que son menos vulnerables a sufrir daño ante una inundación por tsunami, situación que se justifica por el grado de exposición a la profundidad del agua, menor a 1 [m] y a las características estructurales de cada edificación, las que resultaron ser aceptables (Bv = 2-3) para la mayoría de ellas, a pesar de que el entorno ofrece un nivel bajo de protección (Prot $=3-5)$.

Sin embargo, los recintos que presentan un índice de vulnerabilidad mayor son los que están más expuestos a la intrusión del agua, entre 2 y 4 [m] de profundidad y el riesgo de sufrir algún tipo de daño por objetos móviles es muy alto, debido a que en los alrededores se encuentran embarcaciones de gran tamaño.

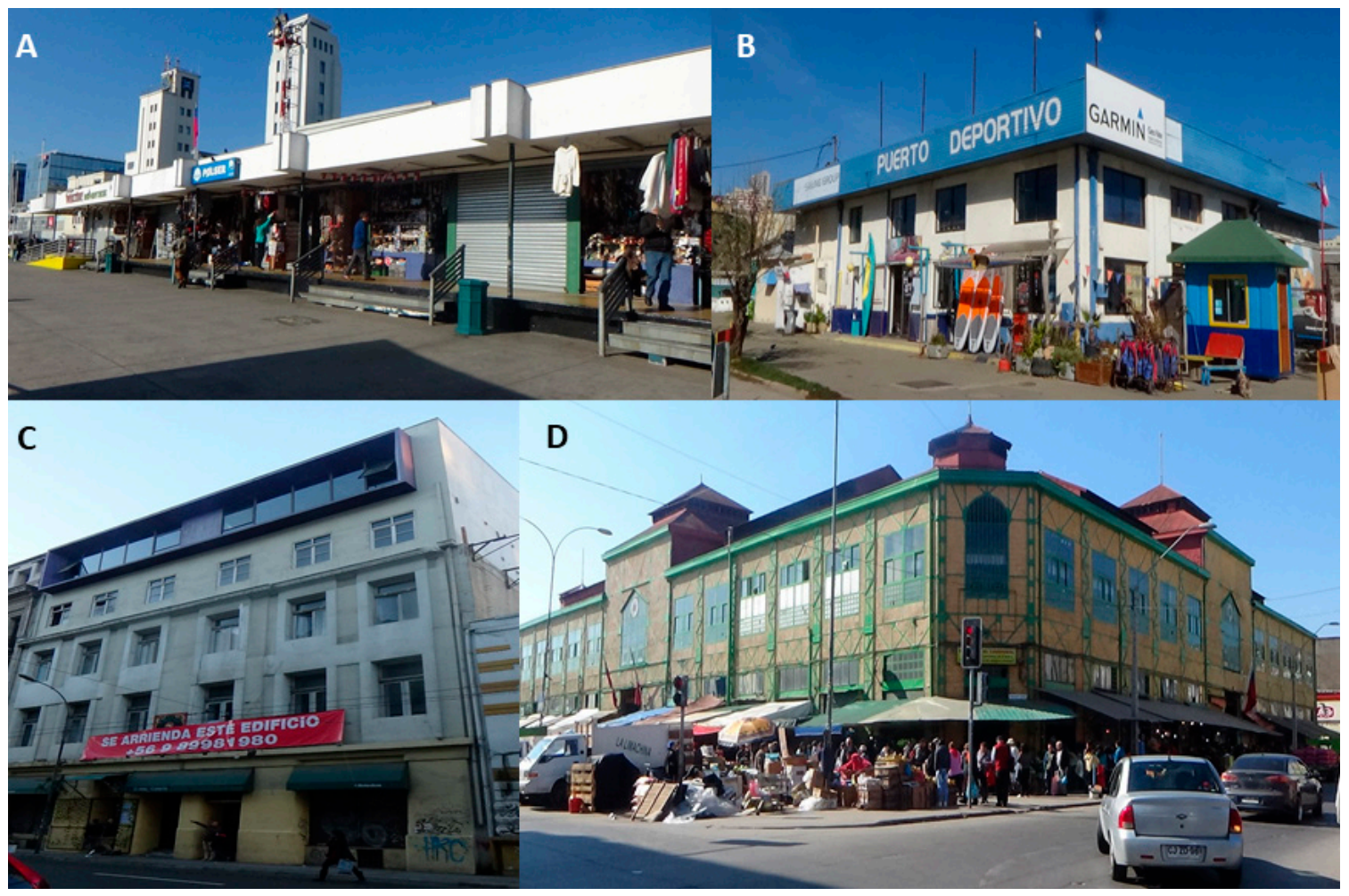

Figura 8. Ejemplos edificaciones asociadas a los atractivos turísticos en el área de inundación por tsunami en Valparaíso. A) y B) ejemplos de estructuras con vulnerabilidad alta. C) vulnerabilidad moderada. D) Vulnerabilidad menor Fuente: Autores, 2019

\section{Servicios}

Con un total de 39 edificaciones levantadas, este es el ítem que arrojó más variabilidad en los índices de vulnerabilidad. Esto se explica porque existen edificios de menor envergadura, centros pequeños de atención o de servicio (cajeros automáticos y estaciones de metro) y otros que se caracterizan por ser edificios muy robustos como son las instituciones bancarias y terminales de pasajeros (ver Figura 9).

Respecto a los establecimientos que prestan servicios de transporte (terminales de pasajeros, estaciones de metro), arrojaron los índices de vulnerabilidad más altos (RVI $=3,7$ ), siendo los establecimientos más vulnerables ante una inundación por tsunami, escenario que se justifica por el grado de exposición a la profundidad del agua y a los atributos estructurales del tipo de construcción, los que son desfavorables ( $\mathrm{Bv}=5$ ). La mayoría de estas estructuras se caracterizan por tener 1 piso, son estructuras compuestas por perfiles metálicos y revestidas con madera y cuentan con fundaciones de tipo superficial. 
Sin embargo, en comparación con los establecimientos que prestan servicios financieros, estos arrojaron los índices de vulnerabilidad más bajos ( $R V I=1-1,7)$, edificios que se caracterizan por ser construidos con hormigón armado o albañilería simple, fundaciones profundas, tienen más de 5 niveles, estado de conservación muy bueno y se encuentran ubicados en zonas donde la profundidad de inundación no supera el metro de altura.

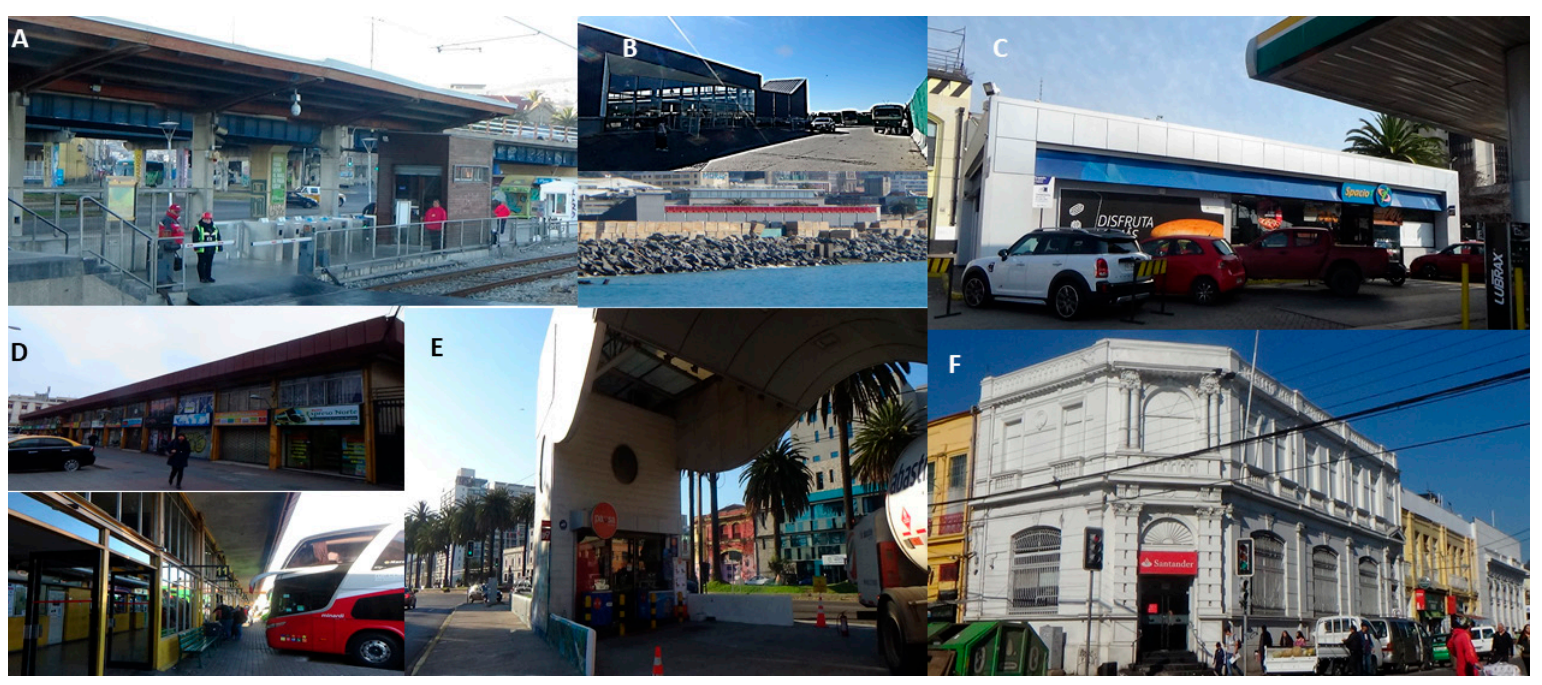

Figura 9. Ejemplo edificaciones asociadas a los servicios turísticos. A) Estación metro-tren, con vulnerabilidad alta. B) Terminal de pasajeros marítimos. Vistas desde tierra y mar. Vulnerabilidad alta. C) Cajero automático dentro de un servicentro. Vulnerabilidad promedio. D) Terminal de pasajeros buses terrestres. Vistas exterior e interior. Vulnerabilidad menor. E) cajero automático - servicio financiero - vulnerabilidad alta. F) Servicio financiero - banco - vulnerabilidad moderada Fuente: Autores, 2019

\section{Edificios con mayor vulnerabilidad}

Dentro de los edificios que muestran mayor vulnerabilidad, se escogieron aquellos con vulnerabilidad mayor a 2,3, correspondientes a 59 estructuras que representan el $26 \%$ del total. En este grupo se encuentran:

- 25 de alimentación (que incluyen todos los de Caleta Portales)

- 4 de alojamiento

- 12 atractivos turísticos

- 18 que albergan servicios

Los factores que describen a una edificación con mayor vulnerabilidad corresponden a edificios de 1 o 2 pisos, así como una alta exposición a objetos móviles. Además, 44 de las 59 edificaciones más vulnerables están en la primera línea litoral. Esto habla de una fuerte intervención de la zona litoral con fines de turismo. Ninguno de los edificios más vulnerables tiene un muro de protección propio para mitigar inundaciones. Dentro de este grupo, se encuentran la mitad de los establecimientos de servicios. Por otro lado, la mitad de las edificaciones más vulnerables son de alimentación.

La vulnerabilidad no es explicada por el material de construcción: 25 de las 59 estructuras más vulnerables fueron construidas con materiales que incrementan su vulnerabilidad (madera, mixto, construcción ligera en general); mientras que 19 edificaciones fueron construidas con materiales robustos (albañilería reforzada, hormigón, etc.). Tampoco por la hidrodinámica, fundaciones, forma y orientación. La mayoría de estas edificaciones tienen un buen estado de preservación, lo que se explica por la necesidad de cuidar la imagen para el turismo.

En cambio, el número de plantas si explica la vulnerabilidad: 30 estructuras son de un piso y 24 de dos pisos, de las 59 estructuras más vulnerables. También lo hace la posición con respecto a la línea costera: 44 de los 59 están en la primera línea litoral y las protecciones del borde costero: 57 de 59 no cuentan con protección natural. Ninguna de estas edificaciones cuenta con un muro de protección. 
Con respecto a la inundación esperada, la mayoría de las 59 edificaciones se encuentra expuesta en menos de 1 metro de inundación. Solo 5 edificios se encuentran en la mayor inundación - frente portuario. Con un piso inundado 44 de 59 edificaciones, mientras que 10 edificios se encuentran en áreas con inundaciones potenciales sobre $4 \mathrm{~m}$.

\section{Edificios con menor vulnerabilidad}

Se escogió como corte vulnerabilidades inferiores a 1,3. Aquí se encuentran 81 edificaciones ( $37 \%$ del total). De estos edificios:

- 14 son de alimentación

- 8 son de alojamiento

- 15 son de atractivos

- 13 son de sistema financiero

Dentro de los edificios menos vulnerables, los atributos físicos que los caracterizan corresponden a edificios de 3 o más pisos, con materialidad robusta como concreto reforzado, fundaciones profundas, y buen estado de preservación. Estas edificaciones se encuentran emplazadas distantes a la primera línea de ciudad, donde se tienen bajas profundidades de inundación.

En efecto, 79 edificios de 81 tienen 3 pisos o más. La mayoría de los materiales con que son construidos son sólidos: 36 edificios de concreto, 2 de ladrillo doble y 35 de ladrillo simple. Ochenta edificaciones cuentan con fundaciones profundas. El $75 \%$ de estas edificaciones tiene un riesgo moderado de sufrir impactos por objetos móviles. También el 75\% de las edificaciones se encuentran sobre el promedio de preservación. En cuanto a la exposición, todos se encuentran en zonas con inundaciones potenciales inferiores a $1 \mathrm{~m}$.

No son variables explicativas para encontrarse dentro de las menos vulnerables: La hidrodinámica, forma y orientación, el número de fila de edificación o posición con respecto al litoral (aun cuando sobre el $50 \%$ se encuentran desde la fila 7 ), la protección y existencia de muros.

\section{DISCUSIÓN}

En las últimas décadas, en la franja costera del país, se están concentrando diversas inversiones tanto para infraestructuras de accesibilidad general (carreteras y autopistas) como construcciones de infraestructuras y equipamientos (alojamientos y marinas, por ejemplo) para el ocio y el turismo, con evidentes repercusiones territoriales, económicas y sociales. El borde costero marítimo y lacustre es el espacio más presionado por este tipo de inversiones, aumentando la demanda estacional de la infraestructura y servicios públicos, generando conflictos con otras actividades productivas (industria, pesca, residencial, silvoagropecuaria, entre otras) y transformando el paisaje y el territorio al introducir estructuras urbanísticas que rompen con la continuidad anterior, que urbanizan el campo y privatizan el territorio, ya sea, a través de urbanizaciones inmobiliario-turísticas concentradas o a través de parcelaciones y construcciones dispersas sobre el espacio rural, lo que se complementa con la expansión de la oferta hotelera y residencial en los núcleos urbanos. Además, los desastres están afectando especialmente los espacios costeros cuando se trata de terremotos que producen tsunamis.

Una mirada territorializada del proceso de desarrollo turístico en el litoral de Chile nos permite observar importantes transformaciones territoriales que están ocurriendo como resultado de su valorización social como espacio de turismo en los últimos años y de la concreción de los intereses turísticos privados y la escasa capacidad de coordinación pública. Entre esas transformaciones se destacan para el objetivo de este artículo las siguientes:

i. Sociales y económicas. Mayor valoración del ocio y del turismo como derecho de la sociedad chilena, valorización turística de espacios deshabitados, masificación de los destinos turísticos (cuantitativa y cualitativamente), tendencia a la monofuncionalidad de ciertas localidades por la especialización en actividades turísticas, presiones del sector inmobiliario y de la construcción sobre la gestión y uso del suelo, estacionalidad de la demanda turística y desincentivo a la inversión, conflictos entre residentes y turistas, control extralocal de los recursos y territorios, desatención ante la acumulación de riesgos antrópicos y naturales en los destinos turísticos.

ii. Territoriales. Densificación poblacional de las zonas costeras y despoblamiento de las 
áreas interiores, concentración de la demanda y la oferta turística en el litoral, concentración de construcciones en el litoral, cambios en la estructura territorial por loteos, urbanizaciones y construcciones espontáneas, segmentación socioeconómica de los destinos turísticos, conflictos de intereses por el uso del suelo en destinos turísticos, urbanización e intervención de paisajes de calidad y desatención de la planificación a los riesgos emergentes como los incendios, tsunamis y marejadas.

iii. Políticas. Fragmentación en la gestión del territorio, descoordinación y fragmentación sectorial, carencia de instrumentos de planificación locales y regionales integrales, paradigma neoliberal hegemónico en la justificación de la gestión de la administración, decisiones políticas de corto plazo frente a la necesidad de ingresos a las arcas municipales, políticas de desarrollo ineficaces por desconocimiento de las características del fenómeno turístico, decisiones que deterioran las áreas costeras porque ignoran su fragilidad medioambiental.

iv. Ambientales. Deterioro de los ecosistemas y del paisaje litoral, agotamiento de los recursos turísticos, deterioro y destrucción del patrimonio cultural en los destinos, las implantaciones turísticas generan importantes externalidades, lo que hace indispensable para la sociedad atenderlas y regularlas, incorporando medidas correctivas en su funcionamiento para armonizar las decisiones con la eficiencia económica, la equidad social y la protección del medio ambiente.

La expansión del turismo en el litoral de Chile muestra que una parte importante del equipamiento turístico sobrepasa los límites normados por los Planes Reguladores locales, teniendo allí una competencia directa la Administración Regional o Nacional, lo que obligaría a hacer funcionar mecanismos de coordinación entre las distintas administraciones (PUCV, 2004). Sin embargo, el Estado carece hoy de una adecuada estructura institucional para coordinar la gestión del turismo y el ordenamiento territorial. A pesar de los avances aún subsiste una alta dispersión de funciones, una falta de autonomía local y regional para dirigir el desarrollo de las actividades territoriales y conflictos de competencia entre Administraciones nacionales, regionales y locales, debido a la superposición de normativas.

Si hay un evento importante de atracción de visitantes a la costa de la ciudad, este ocurre el 31 de diciembre de cada año, momento en el cual se despide el año con las significaciones simbólicas que ello implica y un espectáculo pirotécnico nocturno que coordina a tres comunas del litoral en un recorrido de $30 \mathrm{~km}$ de costa. En Valparaíso se organizan espectáculos musicales en Plaza Sotomayor o las principales plazas del plano de la ciudad próximo a la línea de costa que inician al atardecer y son el preludio de los fuegos artificiales, unos minutos después de las 00:00 hr. Estas actividades se reproducen al mismo tiempo en las localidades turísticas de Viña del Mar, Reñaca y Concón.

La visitación turística y por ocio es la mayor de todo el año, involucra a cerca de un millón de visitantes nacionales y extranjeros. La última semana del año 2017 de acuerdo a un balance realizado por Sernatur Región de Valparaíso, en base a datos entregados por Carabineros, cerca de 683 mil personas llegaron durante el fin de semana al sector costero de la región (Radio Valparaíso: 0201-2018). Esta cifra se repite en los balances de dados a conocer ya sea por la Dirección Nacional de Turismo o por la Cámara Regional de Turismo, en los último diez años. Además, unas 50 embarcaciones menores acogen a visitantes que desean realizar un pequeño recorrido por la bahía y al finalizar el día esperan observar el espectáculo pirotécnico en el mar.

La estrategia de los actores territorializados ya indicada tiene en los actores del mercado una respuesta directa en la instalación de una amplia oferta turística concentrada en localización en el propio atractivo de la ciudad: el área histórico patrimonial. A principios de los años 2000 , y a diferencia de otros destinos, los escasos establecimientos de alojamiento de Valparaíso se dispersan por las áreas llanas de la ciudad utilizando vías secundarias del centro urbano, alejadas de la línea de costa. Las vías de conectividad internas y el valor del suelo son criterios principales de localización. No existen establecimientos de alojamiento en el borde costero o con vista al mar atendiendo a su carácter urbano portuario y a que las tres playas de arena que posee tienen un uso turístico escaso (Figueroa-Sterquel, 2011). La tendencia iniciada en 2003 fue el desarrollo de alojamientos tipo bed and breakfast en sectores históricos altos (cerros) de la ciudad sobre la base de edificios de uso residencial que se re-funcionalizan a alojamiento. Esta tendencia se generalizó 
sobre unas pocas colinas y en el año 2018 los Cerros Alegre y Concepción concentran las ofertas de alojamiento, alimentación y recreación basadas en el principal patrimonio arquitectónico de la ciudad. Otro núcleo se desarrolla en torno al principal mirador de la ciudad en el Cerro Artillería al noroeste. En tanto que la función comercial y lúdica del turismo se emplaza en el sector plano de la ciudad, principalmente el sector financiero, el sector puerto y el sector el Almendral, próximos a la línea de costa. (Figura 10).

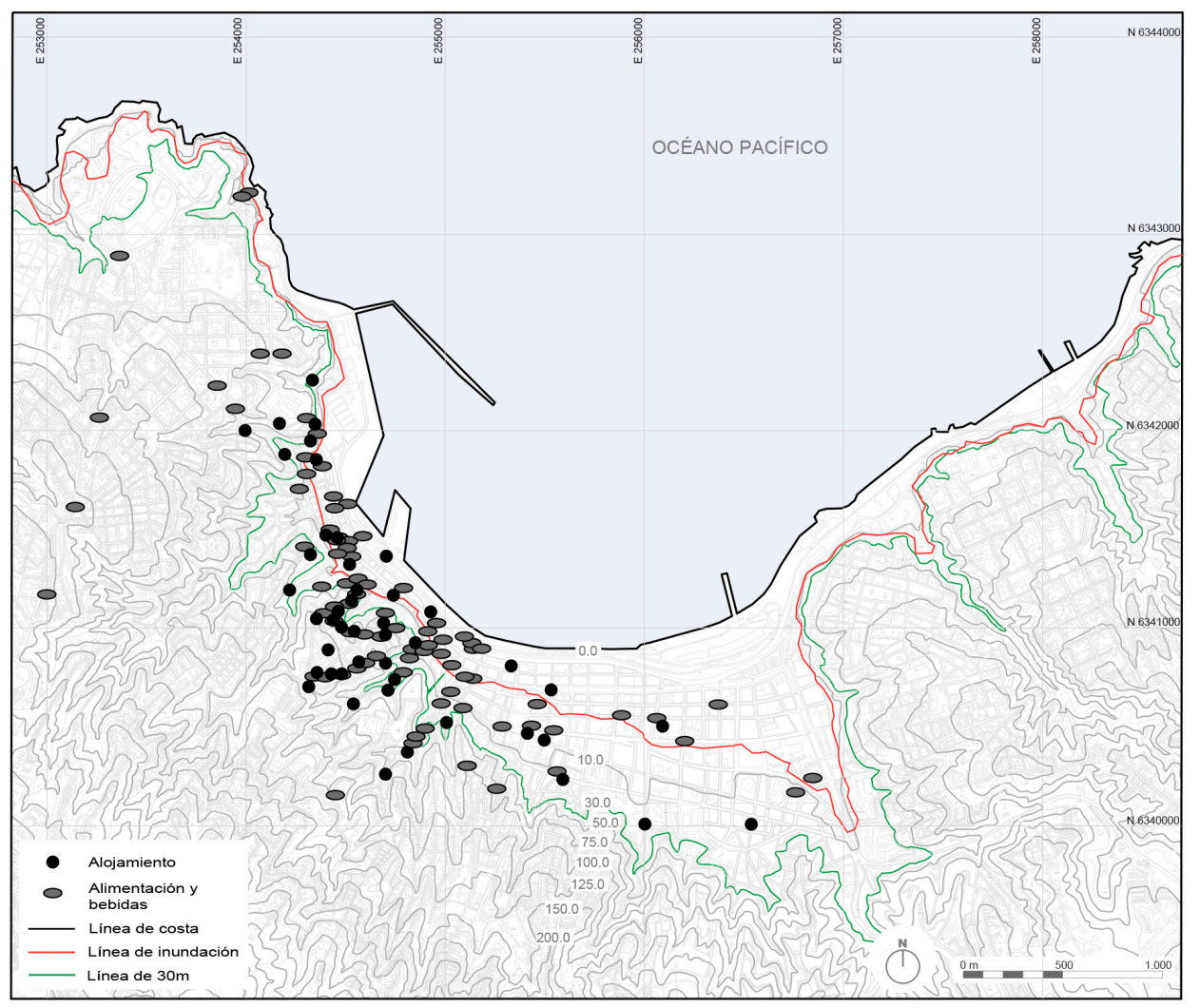

Figura 10. Estrategia de localización de los principales equipamientos turísticos Fuente: Autores, 2019, adaptado de Google Earth 2018

Para los actores territoriales locales, regionales y nacionales de Valparaíso, lo patrimonial se fundamenta en una lógica economicista, teniendo a la comercialización del turismo como elemento articulador, dentro del paradigma desde el cual se ha observado el fenómeno turístico en el transcurso del siglo XX. Así lo evidencian los lineamientos del Plan de Desarrollo Turístico, propuesta estratégica desarrollada por consultores transnacionales españoles para convertir a Valparaíso en destino turístico que ofrece como producto la Ciudad Cultural y Patrimonial. Como lo observamos a partir de los graves efectos del tsunami de febrero de 2010, en la costa centro sur del país, por los graves efectos de los siempre presente incendios urbano-forestales desde el año 2013 en Valparaíso, los aspectos de la vulnerabilidad ante el peligro no están siendo considerados en la gestión de los flujos turísticos. De hecho, la gestión de los flujos no está prevista como competencia u objetivo de los actores territoriales ni comerciales del turismo.

\section{CONCLUSIONES}

En el área de inundación por tsunami en Valparaíso, se encuentran 227 edificaciones asociadas al sector turismo amenazadas. En general muestran bajos índices de vulnerabilidad. Las características que prevalecen corresponden a edificios de dos o más pisos y que tienen una materialidad y sistema constructivos robustos, especialmente en edificaciones asociadas al alojamiento de turistas. Esto se explica en parte por la existencia de una norma sísmica en Chile. Anterior a la norma sísmica, los edificios eran construidos de manera muy robusta en cuanto a su estructura, debido a la alta recurrencia de eventos sísmicos. Además, en Valparaíso se aprecia un bajo uso de los materiales de construcción vulnerables, como la madera. Predominan las edificaciones de hormigón y 
albañilería reforzada. Basado en estos antecedentes, se puede proponer que los edificios turísticos incorporen un sistema de evacuación vertical, en vez de obligar a sus residentes a evacuar y exponerse a la zona de seguridad a través de las vías establecidas las cuales ubican a los pasajeros en un tiempo de máxima exposición durante el proceso de evacuación por tsunami.

Medidas blandas como una correcta y oportuna información en los sectores y edificaciones vulnerables se muestra como una medida fácil de implementar, de bajo costo y con gran impacto en los visitantes, lo que debería orientarse a agregar valor a estos espacios como "lugares seguros" precisamente gracias a la presencia de información de prevención, evacuación y georreferenciación del lugar que se visita.

Analizando los factores de vulnerabilidad levantados en el presente estudio se puede inferir que la vía ferroviaria y las instalaciones portuarias funcionan como barreras de protección. Destaca la vulnerabilidad del Metro de Valparaíso, por el emplazamiento de la línea férrea. Este sistema de transporte es esencial para la conectividad de los habitantes y trabajadores de Valparaíso, por lo que se plantea una relocalización para disminuir su vulnerabilidad.

Por otro lado, la presencia del puerto y la línea férrea ha impedido la aparición de edificaciones turísticas en esta franja. Solo la Caleta Portales y el sector muelle Barón se emplazan entre la línea férrea y la orilla del mar, siendo las zonas con usos turísticos, más expuestas de la zona estudiada, lo que aumenta sustancialmente su vulnerabilidad. Por este motivo se plantea la necesidad de contar con una planificación especial para esta zona debido a que es una zona de alta concurrencia de público.

\section{AGRADECIMIENTOS}

La Empresa Portuaria Valparaíso facilitó los datos de recaladas de cruceros turísticos. El Dr. Marco Cisternas incentivó la concepción de este trabajo en el marco del diplomado "Tsunami en la costa de Latinoamérica: bases científicas, amenaza y vulnerabilidad". Los editores y revisores anónimos con sus comentarios mejoraron sustancialmente este trabajo.

\section{REFERENCIAS}

Alberico, I., Di Fiore, V., Iavarone, R., Petrosino, P., Piemontese, L., Tarallo, D., Punzo, M. \& Marsella, E. (2015). The tsunami vulnerability assessment of urban environments through freely available datasets: The case study of Napoli City (southern Italy). Journal of Marine Science and Engineering, 3(3), 981-1005.

Birkland, T. A., Herabat, P., Little, R. G., \& Wallace, W. A. (2006). The impact of the December 2004 Indian Ocean tsunami on tourism in Thailand. Earthquake Spectra, 22(S3), 889-900.

Canihuante, G. (2006). Turismo en Chile: Paisajes y culturas del pasado, presente y futuro. La Serena, Chile: Fondo Editorial de la Ilustre Municipalidad de la Serena.

Carlsen, J. (2006). Post-tsunami tourism strategies for the Maldives. Tourism Review International, $10(1-2), 69-79$.

Carvajal, M., Cisternas, M., Catalán, P. \& Gorigoitía, N. (2014). Redimensionando el terremoto de 1730 de Chile central, mediante evidencias históricas y geológicas del tsunami resultante. En VIII SLAGFy IV SIAGF. Simposio Latino-Americano de Geografía Física -- Simposio Ibero-Americano de Geografía Física "Riesgos, vulnerabilidades y resiliencia socioambiental para enfrentar los cambios globales", Eje 5 - Desastres naturales, riesgos y vulnerabilidades en América Latina y Península Ibérica. Santiago: Universidad de Chile (pp.1134-1142).

Carvajal, M., Cisternas, M. \& Catalán, P. (2017a). Source of the 1730 Chilean earthquake from historical records: implications for the future tsunami hazard on the coast of Metropolitan Chile. Journal of Geophysical Research: Solid Earth, 122, 3648-366o. doi: 10.1002/2017JBo14063

Carvajal, M., Contreras-López, M., Winckler, P. \& Sepúlveda, I. (2017b). Meteotsunamis Occurring Along the Southwest Coast of South America During an Intense Storm. Pure and Applied Geophysics, 174(8), 3313-3323. doi: 10.1007/sooo24-017-1584-o

CERESIS. (1985). Catálogo de terremotos para América del Sur. Programa para la mitigación de los efectos de los terremotos en la región andina, Proyecto SISRA, Vol. 1-14. Austin: Centro Regional de Sismología para América del Sur.

Contreras-López, M., Igualt, F., Breuer, W. \& Zamora, F. (2019). Vulnerabilidad por tsunamis de servicios de emergencia y establecimientos educacionales en el Gran Valparaíso. En Hidalgo, R. \& Martínez, C. (Eds.) La zona costera en Chile: adaptación y planificación para la resiliencia. Santiago de Chile: GEOLIBRO - Pontificia Universidad Católica de Chile. En prensa. 
Contreras-López, M., Winckler, P., Sepúlveda, I., Andaur-Álvarez, A., Cortés-Molina, M., Guerrero, C. J., Mizobe, C. E., Igualt, F., Breuer, W., Beyá, J. F., Vergara, H. \& Figueroa-Sterquel, R. (2016). Field Survey of the 2015 Chile Tsunami with emphasis on Coastal Wetland and Conservation Areas. Pure and Applied Geophysics, 173(2), 349-367.

Contreras, M. \& Winckler, P. (2013). Pérdidas de vidas, viviendas, infraestructura y embarcaciones por el Tsunami del 27 de febrero de 2010 en la costa central de Chile. Obras y Proyectos, 14, 6-19. doi: 10.4067/So718-28132013000200001

Dominey-Howes, D. \& Papathoma, M. (2007). Validating a tsunami vulnerability assessment model (the PTVA Model) using field data from the 2004 Indian Ocean tsunami. Natural Hazards, 40(1), $113-136$.

Dall'Osso, F., Gonella, M., Gabbianelli, G., Withycombe, G., \& Dominey-Howes, D. (2009). A revised (PTVA) model for assessing the vulnerability of buildings to tsunami damage. Natural Hazards and Earth System Sciences, 9(5), 1557-1565. doi: 10.5194/nhess-9-1557-2009

Dall'Osso, F., Dominey-Howes, D., Tarbotton, C., Summerhayes, S. \& Withycombe, G. (2016). Revision and improvement of the PTVA-3 model for assessing tsunami building vulnerability using "international expert judgment": introducing the PTVA-4 model. Natural Hazards, 83(2), 1229-1256.

Figueroa-Sterquel, R. (2011) Desarrollo turístico y transformaciones territoriales en el litoral central de Chile. Promotores públicos y privados en la producción del espacio turístico. Tesis de Doctorado. Barcelona, España: Universidad de Barcelona.

Fritis, E., Izquierdo, T. \& Abad, M. (2018). Assessing the tsunami building vulnerability PTVA-3 and PTVA-4 models after the $16 \mathrm{~S} 2015$ event in the cities of Coquimbo-La Serena (Chile), Natural Hazards and Earth System Science, 18(6), 1703-1716.

Fritz, H. M., Petroff, C., Catalán, P., Cienfuegos, R., Winckler, P., Kalligeris, N., Weiss, R., Barrientos, S., Meneses, G., Valderas-Bermejo, C., Ebeling, C., Papadopoulos, A., Contreras, M., Almar, R., Domínguez, J. \& Synolakis, C. (2011). Field Survey of the 27 February 2010 Chile Tsunami. Pure and Applied Geophysics, 168(11), 1989-2010.

Goll, F. (1903). Die Erdbeben Chiles: ein Verzeichnis der Erdbeben und Vulkanausbruche in Chile, bis zum Jahre 1879 (Inkl.) nebst einigen Allgemeinen Bemerkungen zu diesen Erdbeben. München: Münchener Geographische Studien.

González, M., \& Figueras, S. (2005). Tsunami de Sumatra del 26 de Diciembre de 2004. Enseñanza de las Ciencias de la Tierra, 13(1), 2-14.

Igualt, F. (2017). Evaluación de vulnerabilidad física y adaptabilidad post-tsunami en Concón, zona central de Chile. AUS [Arquitectura / Urbanismo / Sustentabilidad], 22, 53-58.

Igualt, F., Breuer, W., Winckler, P. \& Contreras-López. M. (2017). Rehabilitación de centros urbanos afectados por el Tsunami 2010 en la Comuna de Pelluhue, Chile. Latin American Journal of Aquatic Research (LAJAR), 43(4), 659-674.

INE. (2017). Estadísticas Demográficas y Vitales. Santiago: Instituto Nacional de Estadística. Disponible en: http://www.ine.cl/estadisticas/demograficas-y-vitales [visitado el 1 de octubre de 2018].

Knafou, R. (1996). Turismo e território: por uma abordagem científica do turismo. En Rodrigues, A.B. (Ed.), Turismo e geografia: reflexões teóricas e enfoques regionais. Sao Paulo: Hucitec (pp. 62-74).

MOP. (2018) Concesión interconexión vial Santiago-Valparaíso-Viña del Mar. Ministerio de Obras Públicas. Informes mensuales. Disponibles en: http://www.concesiones.cl/proyectos/Paginas/detalle_adjudicacion.aspx?item=24 [visitado el 1 de diciembre de 2018].

NGDC. (2011). Observed Water Heights and Computed Travel Times for the March 11, 2011 Japan tsunami. Disponible en: http://itic.ioc-unesco.org/images/stories/ttt_japan2o11.jpg [visitado el 1 de diciembre de 2018].

NOAA. (2017). Tsunami Event Database. National oceanic and atmospheric administration. National Geophysical data center. Disponible en: https://www.ngdc.noaa.gov/nndc/struts/form?$\mathrm{t}=101650 \& \mathrm{~s}=167 \& \mathrm{~d}=166$ [visitado el 1 de octubre de 2018].

Papathoma, M. \& Dominey-Howes, D. (2003). Tsunami vulnerability assessment and its implications for coastal hazard analysis and disaster management planning, Gulf of Corinth, Greece. Natural Hazards and Earth System Science, 3(6), 733-747.

Pontificia Universidad Católica de Valparaíso PUCV. (2004). Plan maestro de desarrollo turístico V $V^{\underline{a}}$ Región de Valparaíso Valparaiso: Instituto de Geografía, Servicio Nacional de Turismo Dirección Regional de Valparaíso. 
Rangel-Buitrago, N., Contreras-López, M., Martínez, C. \& Williams, A. (2018). Can coastal scenery be managed? The Valparaíso region, Chile as a case study, Ocean \& Coastal Management, 163, 383-40o. doi: 10.1016/j.ocecoaman.2018.07.016

Salsman, G. G. (1959). Bulletin №6: The Tsunami of March 9, 1957 as recorded at tide stations. Washington: GPO Technical Bulletin.

Shepard, F. P., Macdonald, G. A. \& Cox, D. C. (1949). The Tsunami of April 1, 1946. Bulletin of the Scripps Institution of Oceanography, 5(6), 391-527.

SHOA. (2000). El maremoto del 22 de mayo de 1960 en las costas de Chile (2da ed.). Valparaíso: Servicio Hidrográfico y Oceanográfico de la Armada de Chile.

SHOA. (2012). TSU-511o Valparaíso - Viña del mar: carta de inundación por tsunami referida al evento del año 1730. Valparaíso: Servicio Hidrográfico y Oceanográfico de la Armada de Chile.

SHOA. (2014). Chile Sea Level Network (observations, marigrams). Valparaíso: Servicio Hidrográfico y Oceanográfico de la Armada de Chile.

Soloviev, S. L. \& Go, C. N. (1984). Catalog of Tsunamis on the Eastern Shore of the Pacific Ocean. Academy of Science of the USSR. $\mathrm{N}^{\circ}$ 5078. Ottawa: Canadian Translation of Fisheries and Aquatic Sciences.

Spaeth, M. G. \& Berkman, S. C. (1967). The Tsunami of March 28, 1964, as recorded at tide stations. Rockville: ESSA Technical Report.

Tammet, T., Luberg, A., \& Järv, P. (2013). Sightsmap: crowd-sourced popularity of the world places. In Information and communication technologies in tourism 2013. Berlin \& Heidelberg: Springer (pp. 314-325).

USDC. (1953). The Tsunami of November 4, 1952, as recorded at tide stations. Special Publication $\mathrm{N}^{\circ} 300$. Washington: U. S. Department of Commerce, Coast and Geodetic Survey.

Vicuña Mackenna, B. (1869). Historia de Valparaíso Tomo I: Crónica política, comercial y pintoresca de su ciudad y de su puerto desde su descubrimiento hasta nuestros días, 1536-1868. Santiago: Imprenta Albión, de Cox y Taylor. 